\title{
Supplemental Material: Bayesian Binomial Mixture Models for Estimating Abundance in Ecological Monitoring Studies
}

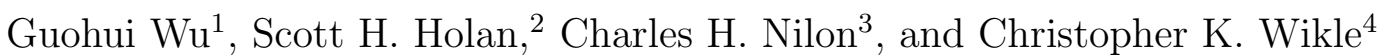

${ }^{1}$ SAS Institute Inc., SAS Campus Drive, Cary, NC 27513

${ }^{2}$ (to whom correspondence should be addressed) Department of Statistics, University of Missouri, 146 Middlebush Hall, Columbia, MO 65211-6100, holans@missouir.edu

${ }^{3}$ Department of Fisheries and Wildlife Sciences, University of Missouri, 302 Anheuser-Busch Natural Resources Building, Columbia, MO 65211-7240

${ }^{4}$ Department of Statistics, University of Missouri, 146 Middlebush Hall, Columbia, MO 65211-6100 


\section{Appendix A: MCMC Sampling Algorithm}

We present the MCMC sampling algorithm for the Bin-CMP model under a given model structure. Specifically, we consider the following model:

$$
\begin{aligned}
y_{i j k} \mid N_{i j}, p_{i j k} & \sim \operatorname{Bin}\left(N_{i j}, p_{i j k}\right), \\
N_{i j} \mid \lambda_{i j}, \nu_{j} & \sim \operatorname{CMP}\left(\lambda_{i j}, \nu_{j}\right), \\
\operatorname{logit}\left(p_{i j k}\right) & =\beta_{1} x_{i j k, 1}+\cdots+\beta_{P} x_{i j k, P}, \\
\log \left(\lambda_{i j}\right) & =\boldsymbol{w}_{i j}^{\prime} \gamma,
\end{aligned}
$$

where $i=1,2, \ldots, G, j=1,2, \ldots, J$, and $k=1,2, \ldots, n_{i j}$. The marginal distribution of observing $\boldsymbol{y}_{i j}$ can be derived by integrating out $N_{i j}$, i.e.,

$$
\left[\boldsymbol{y}_{i j} \mid \mathcal{M}, \boldsymbol{\beta}, \boldsymbol{\gamma}, \nu_{j}\right]=\sum_{N_{i j} \geq y_{i j}^{\max }}^{\infty}\left\{\prod_{k=1}^{n_{i j}} \frac{N_{i j} !}{y_{i j k} !\left(N_{i j}-y_{i j k}\right) !} p_{i j k}^{y_{i j k}}\left(1-p_{i j k}\right)^{N_{i j}-y_{i j k}}\right\} \frac{\lambda_{i j}^{N_{i j}}}{\left(N_{i j} !\right)^{\nu_{j}} Z\left(\lambda_{i j}, \nu_{j}\right)}
$$

where $\boldsymbol{\beta}=\left(\beta_{1}, \ldots, \beta_{P}\right)^{\prime}$ and $y_{i j}^{\max }=\max \left\{\boldsymbol{y}_{i j}\right\}$. Since the calculation of (A.1) involves the summation of infinite series, it suffices to truncate the summation after certain term $N_{i j}^{\max }$. In our case, the choice of $N_{i j}^{\max }$ is based on ecological expertise of the study system. Specifically, for the application to BES data, we set $N_{i j}^{\max }=2 * y_{i j}^{\max }+50$. Additionally, the joint posterior distribution of $\mathcal{M}, \boldsymbol{\beta}, \boldsymbol{\gamma}, \boldsymbol{\nu} \mid \mathbf{Y}$ is given by

$$
\begin{aligned}
{[\mathcal{M}, \boldsymbol{\beta}, \boldsymbol{\gamma}, \boldsymbol{\nu} \mid \mathbf{Y}] } & \propto\left\{\prod_{i=1}^{G} \prod_{j=1}^{J} \mathcal{L}\left(\boldsymbol{y}_{i j} \mid \mathcal{M}, \boldsymbol{\beta}, \boldsymbol{\gamma}, \nu_{j}\right)\right\}\left[\boldsymbol{\beta} \mid \mathcal{M}_{\boldsymbol{\beta}}\right]\left[\boldsymbol{\nu} \mid \mathcal{M}_{\boldsymbol{\nu}}\right]\left[\boldsymbol{\gamma} \mid \mathcal{M}_{\boldsymbol{\gamma}}\right] \\
& \times\left[\mathcal{M}_{\boldsymbol{\beta}}\right]\left[\mathcal{M}_{\boldsymbol{\gamma}}\right]\left[\mathcal{M}_{\boldsymbol{\nu}}\right]
\end{aligned}
$$

In what follows, let $[X \mid \cdot]$ denote the full conditional distribution of $X$ given the data and other parameters in the model. In addition, we define $\mathbf{1}(X \geq x)$ as an indicator function that takes a value of 1 if the condition $X \geq x$ is met and 0 otherwise. 


\section{A.1 Update $N_{i j}$}

For $i=1,2, \ldots, G$ and $j=1,2, \ldots, J$, the full conditional distribution of $N_{i j},\left[N_{i j} \mid \cdot\right]$, is

$$
\begin{aligned}
{\left[N_{i j} \mid \cdot\right] } & \propto\left\{\prod_{k=1}^{n_{i j}}\left[y_{i j k} \mid N_{i j}, p_{i j k}\right]\right\}\left[N_{i j} \mid \lambda_{i j}, \nu_{j}\right] \mathbf{1}\left(N_{i j} \geq y_{i j}^{\max }\right) \\
& \propto\left\{\prod_{k=1}^{n_{i j}} \frac{N_{i j} !}{\left(N_{i j}-y_{i j k}\right) !}\left(1-p_{i j k}\right)^{N_{i j}-y_{i j k}}\right\} \frac{\lambda_{i j}^{N_{i j}}}{\left(N_{i j} !\right)^{\nu_{j}} Z\left(\lambda_{i j}, \nu_{j}\right)} \mathbf{1}\left(N_{i j} \geq y_{i j}^{\max }\right) \\
& \propto\left\{\lambda_{i j} \prod_{k=1}^{n_{i j}}\left(1-p_{i j k}\right)\right\} \frac{1}{\left(N_{i j} !\right)^{\nu_{j}}} \prod_{k=1}^{n_{i j}} \frac{N_{i j} !}{\left(N_{i j}-y_{i j k}\right) !} \mathbf{1}\left(N_{i j} \geq y_{i j}^{\max }\right) .
\end{aligned}
$$

Given $N_{i j} \geq y_{i j}^{\max }$, taking the logarithm of A.2 yields

$$
\begin{aligned}
\log \left\{\left[N_{i j} \mid \cdot\right]\right\} & =\text { const }+N_{i j}\left\{\log \left(\lambda_{i j}\right)+\sum_{k=1}^{n_{i j}} \log \left(1-p_{i j k}\right)\right\}+\left(n_{i j}-\nu_{j}\right) \log \left(N_{i j} !\right) \\
& -\sum_{k=1}^{n_{i j}} \log \left\{\left(N_{i j}-y_{i j k}\right) !\right\},
\end{aligned}
$$

where const denotes a constant value. Note that each $N_{i j}$ is a discrete variable and we can obtain their independent posterior samples using a "grid search" updating scheme following the steps outlined below:

Step 1: Determine the lower and upper bound for each $N_{i j}$ and denote them as $N_{i j}^{\min }$ and $N_{i j}^{\max }$ respectively, where $N_{i j}^{\min } \geq y_{i j}^{\max }$.

Step 2: For each $N_{i j} \in\left\{N_{i j}^{\min }, N_{i j}^{\min }+1, \ldots, N_{i j}^{\max }\right\}$, evaluate A.3 and denote the resulting values as $\left\{q_{i j}^{1}, q_{i j}^{2}, \ldots, q_{i j}^{L}\right\}$, where $L=N_{i j}^{\max }-N_{i j}^{\min }+1$.

Step 3: Randomly draw $N_{i j}$ from $\left\{N_{i j}^{\min }, N_{i j}^{\min }+1, \ldots, N_{i j}^{\max }\right\}$ using $\left\{q_{i j}^{1}, q_{i j}^{2}, \ldots, q_{i j}^{L}\right\}$ as sampling weights.

\section{A.2 Update $\nu$}

The parameter $\boldsymbol{\nu}$ is updated using the Metropolis-Hastings algorithm. Given the model structure $\mathcal{M}_{\boldsymbol{\nu}}$, let $\nu_{j}=\nu_{h}^{(k)}, \forall j \in T_{h} \subset \mathcal{M}_{\nu}$ be the value of dispersion parameters in the 
grouping $T_{h}$ at the $k$-th iteration. The algorithm reads:

Step 1: At iteration $k+1$, propose $\nu_{h}^{\star} \sim \operatorname{Unif}\left(\nu_{h}^{(k)}-c_{\nu^{h}}, \nu_{h}^{(k)}+c_{\nu^{h}}\right)$, where $c_{\nu^{h}}$ is a tuning parameter.

Step 2: Calculate the acceptance ratio $r$ as

$$
r=\frac{\prod_{j \in T_{h}} \prod_{i=1}^{G}\left[\boldsymbol{y}_{i j} \mid \mathcal{M}_{\boldsymbol{\beta}}, \boldsymbol{\beta}, \mathcal{M}_{\boldsymbol{\gamma}}, \boldsymbol{\gamma}, \mathcal{M}_{\left.\boldsymbol{\nu}, \nu_{h}^{\star}\right]}\right.}{\prod_{j \in T_{h}} \prod_{i=1}^{G}\left[\boldsymbol{y}_{i j} \mid \mathcal{M}_{\boldsymbol{\beta}}, \boldsymbol{\beta}, \mathcal{M}_{\boldsymbol{\gamma}}, \boldsymbol{\gamma}, \mathcal{M}_{\boldsymbol{\nu}}, \nu_{h}^{(k)}\right]}
$$

Step 3: Accept $\nu_{h}^{(k+1)}=\nu_{h}^{\star}$ with probability of $\min (1, r)$.

Step 4: Repeat Steps 1-3 for all groupings $T_{h} \subset \mathcal{M}_{\nu}$.

\section{A.3 Update $\gamma$}

Given the model structure $\mathcal{M}_{\boldsymbol{\gamma}}$, updating $\boldsymbol{\gamma}$ requires the Metropolis-Hastings algorithm. The sampling algorithm proceeds as below:

Step 1: At iteration $k+1$, propose $\boldsymbol{\gamma}^{\star}$ according to $\boldsymbol{\gamma}^{\star} \sim N\left(\boldsymbol{\gamma}^{(k)}, \boldsymbol{c}_{\gamma}\right)$, where $\boldsymbol{c}_{\gamma}$ is a vector of tuning parameters.

Step 2: Calculate the acceptance ratio $r$ as

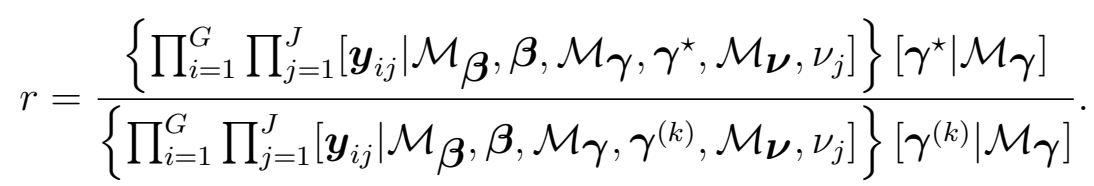

Step 3: Accept $\gamma^{(k+1)}=\gamma^{\star}$ with probability of $\min (1, r)$.

\section{A.4 Update $\beta$}

Given the model structure $\mathcal{M}_{\boldsymbol{\beta}}$, updating $\boldsymbol{\beta}$ requires the Metropolis-Hastings algorithm. The sampling algorithm proceeds as follows: 
Step 1: At iteration $k+1$, propose $\boldsymbol{\beta}^{\star}$ according to $\boldsymbol{\beta}^{\star} \sim N\left(\boldsymbol{\beta}^{(k)}, \boldsymbol{c}_{\beta}\right)$, where $\boldsymbol{c}_{\beta}$ is a vector of tuning parameters.

Step 2: Calculate the acceptance ratio $r$ as

$$
r=\frac{\left\{\prod_{i=1}^{G} \prod_{j=1}^{J}\left[\boldsymbol{y}_{i j} \mid \mathcal{M}_{\boldsymbol{\beta}}, \boldsymbol{\beta}^{\star}, \mathcal{M}_{\boldsymbol{\gamma}}, \boldsymbol{\gamma}, \mathcal{M}_{\boldsymbol{\nu}}, \nu_{j}\right]\right\}\left[\boldsymbol{\beta}^{\star} \mid \mathcal{M}_{\boldsymbol{\beta}}\right]}{\left\{\prod_{i=1}^{G} \prod_{j=1}^{J}\left[\boldsymbol{y}_{i j} \mid \mathcal{M}_{\boldsymbol{\beta}}, \boldsymbol{\beta}^{(k)}, \mathcal{M}_{\boldsymbol{\gamma}}, \boldsymbol{\gamma}, \mathcal{M}_{\boldsymbol{\nu}}, \nu_{j}\right]\right\}\left[\boldsymbol{\beta}^{(k)} \mid \mathcal{M}_{\boldsymbol{\beta}}\right]} .
$$

Step 3: Accept $\boldsymbol{\beta}^{(k+1)}=\boldsymbol{\beta}^{\star}$ with probability of $\min (1, r)$.

\section{Appendix B: Computation Time}

An important aspect of the Bin-CMP models implemented is the computation time needed.

Of the three models considered, $\mathbf{M} 1$ and $\mathbf{M} 2$ take advantage of RJMCMC for variable selection in the model for the intensity function. As such, these two models are more computationally intensive relative to model $\mathbf{M}$ 3. Therefore, computation times for $\mathbf{M} 1$ and M2 exceed the computation time needed for implementing M3 and thus the approximate computation time for M3 is not provided.

In general, the computation time for model implementation will depend on the hardware, programming platform, and how computational parallelism is handled. Because these factors will cause variability in the exact computation time, we provide approximate computation times for models M1 and M2 using 10 knot points (in the spatial case) and note that these computation times may vary depending on the particular implementation with additional efforts in efficient computation leading to reduce computation times. The computations for both models were executed on a cluster consisting of $12 \mathrm{CPU}$ cores (CPU: Intel(R) Xeon(R) X5675 3.07 GHz) with the number of CPUs utilized being two. The computation time for M1 was approximately 39 hours whereas for M2 the approximate computation time was 22 hours. Both models were coded using the $\mathrm{C}$ programming language with an $\mathrm{R}$ executable interface and consisted of 120,000 iterations. 


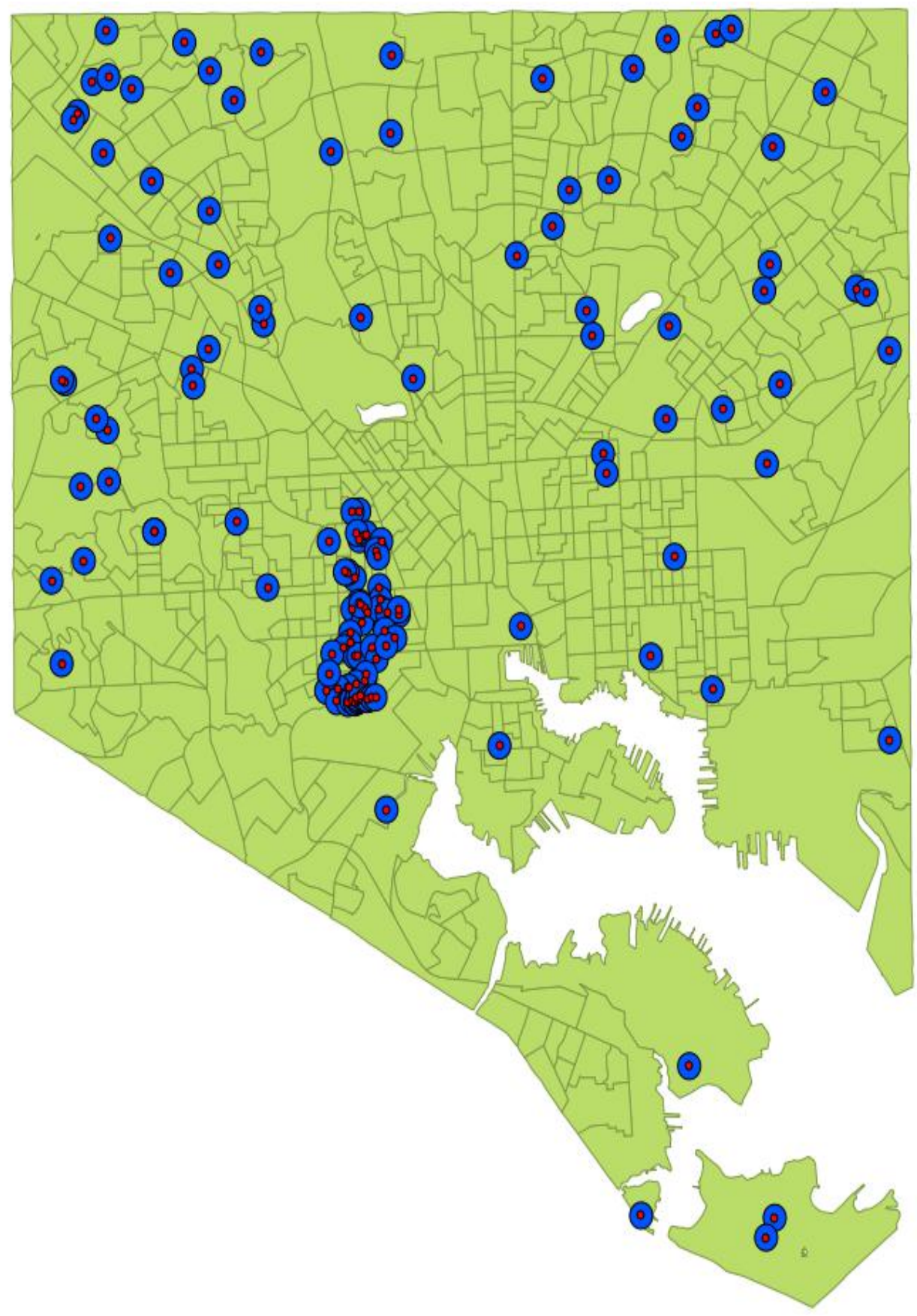

Figure 1: Plot of Baltimore Ecosystem Study (BES) locations. The areal units are census tract block groups, the blue circles are the UFORE plots, and the red circles are the $200 \mathrm{~m}$ radius plots. 


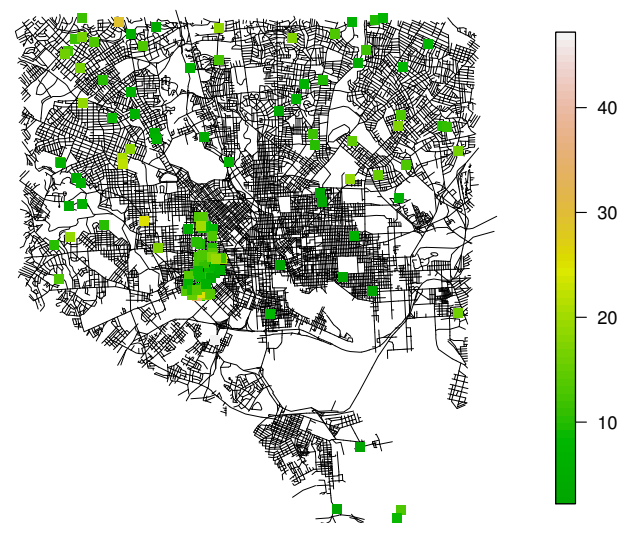

(a) 2005

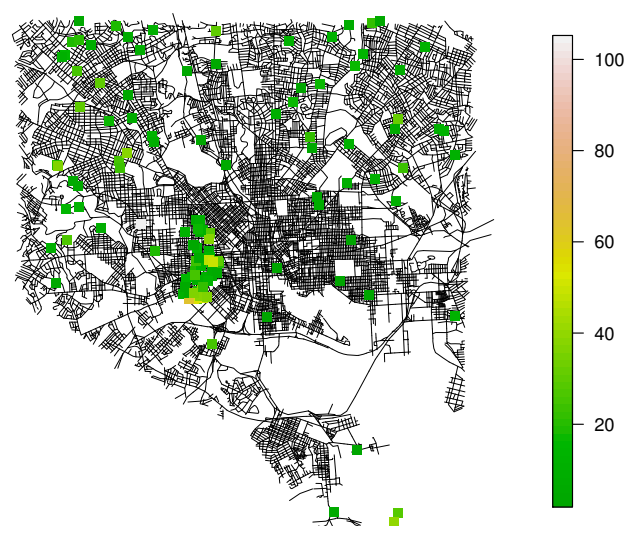

(c) 2006

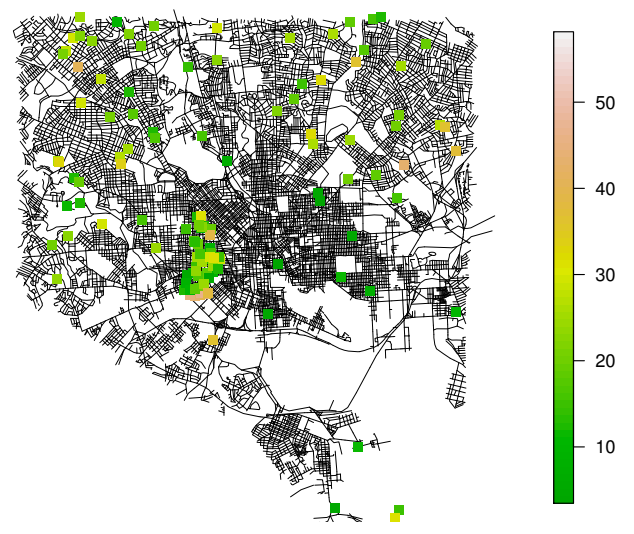

(e) 2007

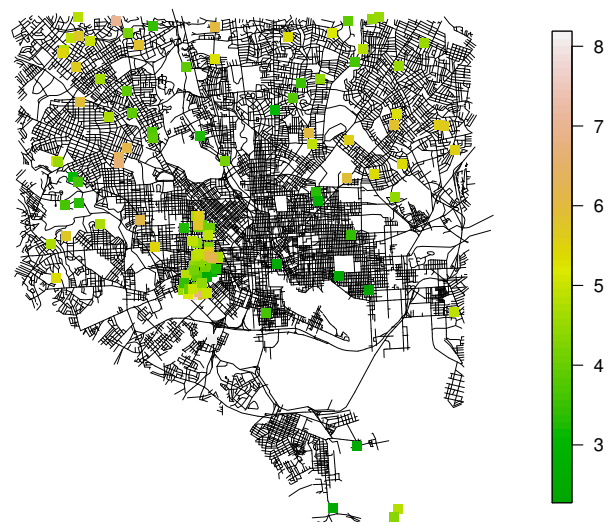

(b) 2005

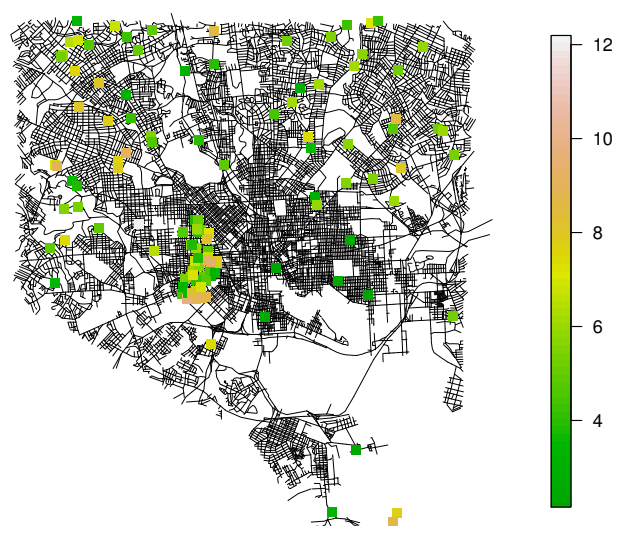

(d) 2006

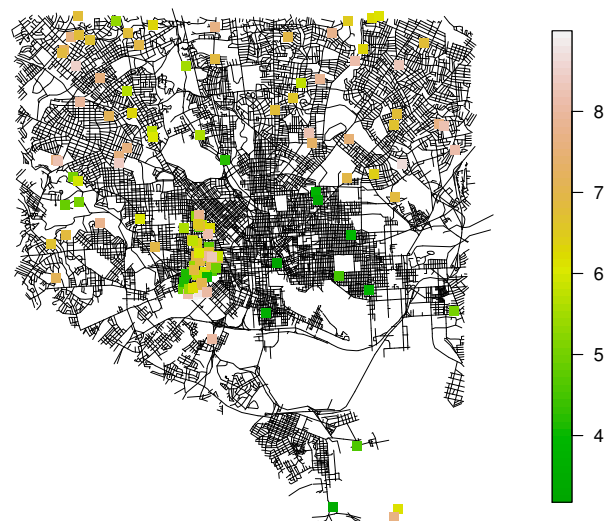

(f) 2007

Figure 2: Plots of posterior mean (1st column) and standard deviation (2nd column) of abundance estimates for 2005-2007 for the Bin-CMP model assuming the posterior mode model for M2 (see Section 6 - main text). 


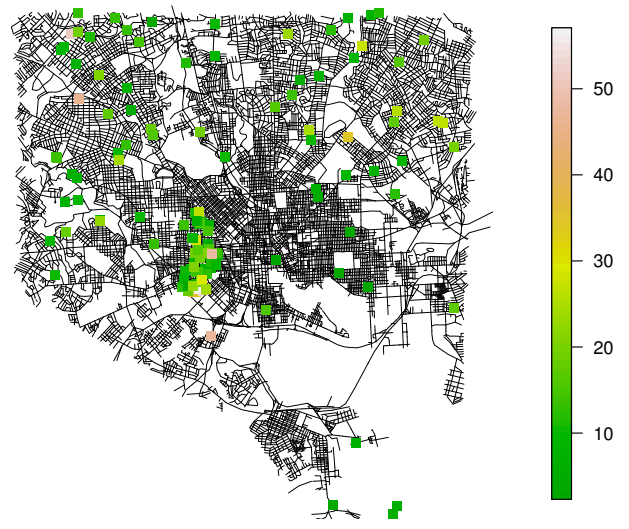

(a) 2008

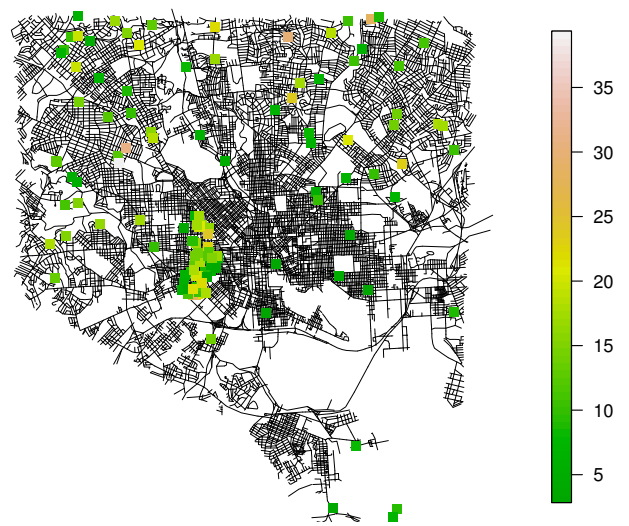

(c) 2009

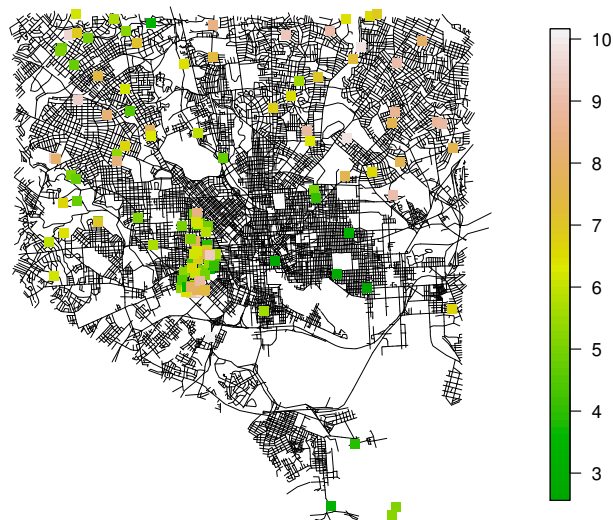

(b) 2008

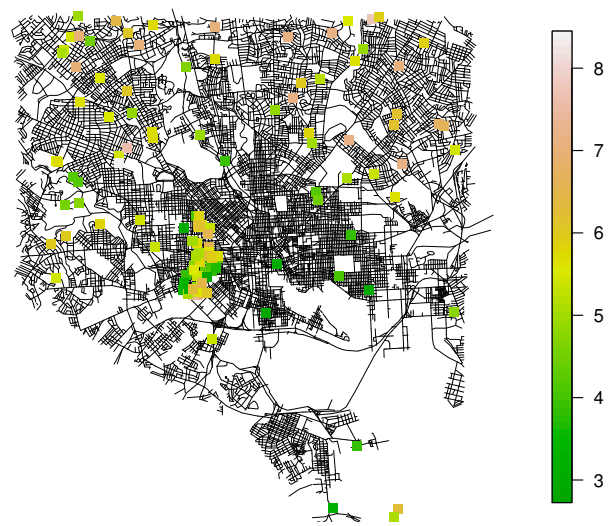

(d) 2009

Figure 3: Plots of posterior mean (1st column) and standard deviation (2nd column) of abundance estimates for 2008-2009 for the Bin-CMP model assuming the posterior mode model for M2 (see Section 6 - main text). 